\title{
Der Raum der primitiven Ideale von endlichen Erweiterungen lokalkompakter Gruppen
}

\author{
Von \\ Detren Poguntke
}

In [8] haben C. C. Moore und J. Rosenberg die Frage untersucht, für welche lokalkompakten Gruppen $G$ der topologische Raum Prim $(G)$ der primitiven Ideale in der einhüllenden $C^{*}$-Algebra $C^{*}(G)$ von $L^{1}(G)$ das $T_{1}$-Axiom erfüllt (d.h. primitive Ideale sind maximal). Für zusammenhängende lokalkompakte Gruppen $G$ wurde die gestellte Frage vollständig gelöst: $\operatorname{Prim}(G)$ ist genau dann ein $T_{1}$-Raum, wenn $G$ projektiver Limes von Lieschen Gruppen ist, für welche die adjungierte Darstellung auf dem Radikal ihrer Lieschen Algebra eine „distale“ Operation ist. Bei der Untersuchung fastzusammenhängender (die Zusammenhangskomponente des Einselementes ist cokompakt) Gruppen und diskreter Gruppen stießen Moore und Rosenberg auf die Frage, wie sich die $T_{1}$-Eigenschaft bei endlichen Erweiterungen verhält. Sie bewiesen den folgenden

Satz. Sei $N$ ein offener Normalteiler von endlichem Index in der (separablen) lokalkompakten Gruppe G. Dann gilt:

(i) Ist $\operatorname{Prim}(G)$ ein $T_{1}-$ Raum, so auch $\operatorname{Prim}(N)$.

(ii) Ist $G / N$ auflösbar und $\operatorname{Prim}(N)$ ein $T_{1}$-Raum, so auch $\operatorname{Prim}(G)$.

(iii) Ist $G / N$ auflösbar und jeder Punkt in Prim $(N)$ lokal abgeschlossen, so gilt dies auch für Prim (G).

Die Voraussetzung der Separabilität wurde in Klammern gesetzt, da sie in [8] gemacht wurde, sich aber als nicht notwendig erweist. Moore und Rosenberg stellten die Frage, ob man auf die Voraussetzung der Auflösbarkeit in (ii) und (iii) verzichten kann. Es ist das Ziel der vorliegenden Arbeit, zu zeigen, daß man dieses in der Tat tun kann.

In [8] wurde bereits ausgeführt, daß man dazu nur den folgenden Satz zu beweisen braucht.

Satz 1. Sei $N$ offener Normalteiler von endlichem Index in der lokalkompakten Gruppe $G$. Es sei $\lambda$ eine stetige irreduzible unitäre Darstellung von $N$. Sind $\sigma$ und $\tau$ stetige irreduzible unitäre Darstellungen von $G$, deren Einschränkungen auf $N$ die Dar- 
stellung $\lambda$ enthalten, und ist $\sigma$ schwach in $\tau$ enthalten (in Zeichen: $\sigma \ll \tau$ ), so sind $\sigma$ und $\tau$ schwach äquivalent.

Der Vollständigkeit halber wollen wir die Argumentation aus [8] wiederholen und zeigen, daß man aus Satz 1 erhält:

Satz 2. Sei $N$ ein offener Normalteiler von endlichem Index in der lokalkompakten Gruppe G. Ist Prim $(N)$ ein $T_{1}$-Raum, so auch Prim $(G)$.

Bemerkung. Die Verallgemeinerung von (iii) auf den nicht-auflösbaren Fall mit Hilfe von Satz 1 ist ein wenig komplizierter als (ii), aber mit der in [8] dargestellten Argumentation ohne Schwierigkeit durchzuführen.

Beweis von Satz 2. Offenbar genügt es zu zeigen: Sind $\alpha$ und $\beta$ zwei stetige irreduzible unitäre Darstellungen von $G$ mit $\alpha \ll \beta$, so sind $\alpha$ und $\beta$ schwach äquivalent. Nach Korollar 2 in [9] zerfallen die Einschränkungen $\left.\alpha\right|_{N}$ und $\left.\beta\right|_{N}$ in endliche Summen irreduzibler Darstellungen von $N$. Nach [2] ist $\left.\left.\alpha\right|_{N} \ll \beta\right|_{N}$. Es gibt folglich eine irreduzible Teildarstellung $\alpha^{\prime}$ von $\left.\alpha\right|_{N}$ und eine irreduzible Teildarstellung $\beta^{\prime}$ von $\left.\beta\right|_{N}$ mit $\alpha^{\prime} \ll \beta^{\prime}$. Da Prim $(N)$ nach Voraussetzung ein $T_{1}$-Raum ist, sind $\alpha^{\prime}$ und $\beta^{\prime}$ schwach äquivalent. Also sind auch die induzierten Darstellungen ind $\alpha_{N \uparrow G}^{\prime}$ und ind $\beta^{\prime}$ schwach äquivalent (die Stetigkeit der Induktion ist in [3] zwar nur für den separablen Fall bewiesen, doch im Falle endlicher Erweiterungen kommt man ohne Separabilität aus, da man den Kern einer induzierten Darstellung (in der $C^{*}$-Hülle) mit Hilfe des Kernes der Ausgangsdarstellung explizit angeben kann). Nun ist $\beta$ eine irreduzible Teildarstellung von ind $\beta^{\prime}$ und daher schwach enthalten in einer irreduziblen Teil$N \uparrow G$

darstellung $\gamma$ von ind $\alpha^{\prime}$. Wir erhalten $\alpha \ll \beta \ll \gamma$. Satz 1 liefert $\gamma \ll \alpha$. Also sind $\alpha$ und $\beta$ schwach äquivalent.

Der Beweis von Satz 1 verläuft folgendermaßen. Mit Hilfe von

Lemma 1. Sei $N$ ein offener Normalteiler von endlichem Index in der lokalkompakten Gruppe $G$. Es sei $\lambda$ eine stetige irreduzible unitäre Darstellung von $N$. $G_{\lambda}$ sei der Stabilisator von $\lambda$ im unitären Dual $N$ von $N$, d.h. $g \in G_{\lambda} \Leftrightarrow$ die Darstellungen $\lambda$ und $n \mapsto \lambda\left(g n g^{-1}\right)$ von $N$ sind unitär äquivalent. Es sei $\gamma$ eine stetige irreduzible unitäre Darstellung von $G_{\lambda}$, deren Einschränkung auf $N$ die Darstellung $\lambda$ enthält. Dann gelten

(i) $\left.\gamma\right|_{N}$ ist ein (endliches) Vielfaches von $\lambda$.

(ii) ind $\gamma$ ist irreduzibel.

$G_{2} \uparrow G$

führen wir Satz 1 auf den Fall $G=G_{\lambda}$ zurück. Unter Verwendung der Mackeyschen Theorie der Erweiterungen reduzieren wir dann unser Problem auf den Fall, daß $\lambda$ auf $G$ fortsetzbar ist. Dann kennt man die über $\lambda$ liegenden irreduziblen Darstellungen genau. Es gilt nämlich 
Lemma 2. Sei $N$ ein offener Normalteiler von endlichem Index in der lokalkompakten Gruppe $G$. Es sei $\pi$ eine stetige unitäre Darstellung von $G$. Die Einschränkung von $\pi$ auf $N$ sei irreduzibel. Ist dann $\pi^{\prime}$ eine stetige irreduzible unitäre Darstellung von $G$ derart, daß $\left.\pi\right|_{N}$ in $\left.\pi^{\prime}\right|_{N}$ enthalten ist, so gibt es eine irreduzible unitäre Darstellung $\alpha$ von $G / N$ mit $\pi^{\prime} \sim \pi \otimes \propto(\sim$ bezeichnet unitäre Äquivalenz $)$. $\propto$ ist bis auf unitäre Äquivalenz eindeutig bestimmt.

Das folgende Lemma beinhaltet dann den wesentlichen Schritt zur Lösung unseres Problems, d.h. zum Beweis von Satz 1.

Lemma 3. $G, N$, $\pi$ seien wie in Lemma 2. Ferner sei $\varrho$ eine irreduzible unitäre Darstellung von $G / N$. Ist $\pi \ll \pi \otimes \varrho$ und bezeichnet $\varrho^{*}$ die konjugierte Darstellung $z u \varrho$, so gilt $\pi \otimes \varrho^{*} \ll \pi$.

Korollar zu Lemma 3. $G, N, \pi$ und @ seien wie in Lemma 2. Ist $\pi \ll \pi \otimes \varrho$, so sind $\pi$ und $\pi \otimes \varrho$ schwach äquivalent.

Wir beweisen nun die obigen Hilfssätze in der angegebenen Reihenfolge und geben danach einen Beweis für Satz 1.

Beweis von Lemma 1 . Unter dem Frobeniusschen Reziprozitätssatz wollen wir in dieser Arbeit die ,globale" Fassung im Sinne von [7] verstehen, d.h. die Isomorphie von $\operatorname{Hom}_{N}\left(\alpha,\left.\beta\right|_{N}\right)$ und $\operatorname{Hom}_{G}($ ind $\alpha, \beta)$ für unitäre Darstellungen $\alpha$ und $\beta$ von $N$ bzw. G. Für (normale) offene Untergruppen $N$ von endlichem Index in $G$ sind die angegebenen Räume von Verkettungsoperatoren tatsächlich isomorph, wie der Beweis in [7] lehrt (man kann in diesem Fall auf Separabilität verzichten), und die in [7] gegebene Definition der induzierten Darstellung fällt mit der üblichen zusammen. ad (i): Nach dem Frobeniusschen Satz ist $\gamma$ in ind $\lambda$ enthalten. Die Einschränkung $N \uparrow G_{\lambda}$

letzterer Darstellung auf $N$ ist aber offenbar ein Vielfaches von $\lambda$. ad(ii): Es sei $m$ die Vielfach heit von $\lambda$ in $\left.\gamma\right|_{N}$, d.h. $\left.\gamma\right|_{N} \sim m \lambda$. Wir setzen $\delta=$ ind $_{G_{\lambda} \star G}$. Es ist zu zeigen, daß $\operatorname{Hom}_{G}(\delta, \delta)$ eindimensional ist oder, nach Frobenius, daß $\gamma$ genau einmal in $\left.\delta\right|_{G_{\lambda}}$ enthalten ist. Ist $G=\bigcup_{i=1}^{\pi} G_{\lambda} x_{i}$ eine Zerlegung von $G\left(x_{1}=e\right)$ und $\lambda^{i}$ definiert durch $\lambda^{i}(y)=\lambda\left(x_{i} y x_{i}^{-1}\right), y \in N$, so sieht man leicht, daß $\left.\delta\right|_{N}$ die orthogonale Summe der $m \lambda_{i}$ ist, und diese Zerlegung von $\left.\delta\right|_{N}$ ist die eindeutige Zerlegung in isotype Komponenten. Der zu $m \lambda$ gehörige Teilraum des Darstellungsraumes von $\delta$ ist $G_{\lambda}$-stabil und $G_{\lambda}$ wirkt dort wie $\gamma$; sein orthogonales Komplement kann offenbar $\gamma$ nicht enthalten, da die $\lambda_{i} i$ für $i>\left.1 \mathrm{zu} \gamma\right|_{N}$ disjunkt sind.

Beweis von Lemma 2. Nach Voraussetzung ist $\pi^{\prime}$ auf Grund des Frobeniusschen Reziprozitätssatzes in ind $\left(\left.\pi\right|_{N}\right)$ enthalten. Nun kann man die induzierte Darstellung einer fortsetzbaren Darstellung sofort „berechnen": ind $\left(\left.\pi\right|_{N}\right)$ ist unitär äquivalent zu dem Tensorprodukt aus $\pi$ und der regulären Darstellung von $G / N$. Dieses Tensorprodukt kann man leicht in irreduzible Komponenten zerlegen, und es zeigt sich, daß 
$\pi^{\prime}$ unitär äquivalent zu einer Darstellung der angegebenen Form ist. Nun zur Eindeutigkeit: Sei $\pi \otimes \alpha \sim \pi \otimes \beta$. Wegen $\operatorname{Hom}_{G}(\pi \otimes \alpha, \pi \otimes \beta) \cong \operatorname{Hom}_{G}\left(\pi, \pi \otimes \beta \otimes \alpha^{*}\right)$ ist $\pi$ in $\pi \otimes \beta \otimes \alpha^{*}$ enthalten. Sei $0 \neq T \in \operatorname{Hom}_{G}\left(\pi, \pi \otimes \beta \otimes \alpha^{*}\right)$ und sei $v_{1}, \ldots, v_{s}$ eine Basis des Darstellungsraumes von $\beta \otimes \alpha^{*}$. Da $\left.\pi\right|_{N}$ irreduzibel ist und $\left.\pi \otimes \beta \otimes \alpha^{*}\right|_{N}$ ein Vielfaches dieser irreduziblen Darstellung ist, gibt es komplexe Zahlen $z_{1}, \ldots, z_{\delta}$ so, daß für jedes $x$ aus dem Darstellungsraum von $\pi$ gilt:

$$
T x=\sum_{i=1}^{s} z_{i} x \otimes v_{i}=x \otimes \sum_{i=1}^{s} z_{i} v_{i} . \text { Da } T \neq 0 \text {, ist } v:=\sum_{i=1}^{s} z_{i} v_{i} \neq 0 .
$$

Aus der Tatsache, daß $T$ ein Verkettungsoperator ist, ergibt sich, daß $v$ bei allen $\left(\beta \otimes \alpha^{*}\right)(g)$ fest bleibt. Also ist die triviale Darstellung in $\beta \otimes \alpha^{*}$ enthalten, und $\alpha$ und $\beta$ sind demnach unitär äquivalent.

Beweis von Lemma 3. Sei $\mathscr{H}$ bzw. $V$ der Darstellungsraum von $\pi$ bzw. $\varrho$. Wir bezeichnen im folgenden korrespondierende Darstellungen von $G$ und $C^{*}(G)$ mit demselben Buchstaben. Unter dem Kern einer Darstellung verstehen wir stets den Kern in $C^{*}(G)$. Wie man leicht sieht, ist die $C^{*}$-Algebra $\mathscr{A}:=(\pi \otimes \varrho)\left(C^{*}(G)\right)$ enthalten in der $C^{*}$-Algebra $\mathscr{B}:=\pi\left(C^{*}(G)\right) \otimes \mathscr{B}(V)$ wobei $\mathscr{B}(V)$ die Algebra aller linearen Operatoren auf dem endlichdimensionalen Raum $V$ bezeichnet. Nach $\nabla$ oraussetzung ist $\pi$ schwach in $\pi \otimes \varrho$ enthalten, d.h. Kern $\pi \supseteqq \operatorname{Kern} \pi \otimes \varrho$. Es gibt daher genau eine stetige irreduzible ${ }^{*}$-Darstellung $\pi^{\prime}$ von $\mathscr{A}$ in $\mathscr{H}$ mit $\pi^{\prime} \circ \pi \otimes \varrho=\pi$. Nach 2.10 .2 aus [1], p. 51, läßt sich $\pi^{\prime}$ zu einer stetigen irreduziblen *.Darstellung $\tau$ von $\mathscr{B}$ in einem Hilbertschen Raum $\mathscr{K}$ erweitern, d.h. die Einschränkung $\left.\tau\right|_{\mathscr{A}}$ enthält eine zu $\pi^{\prime}$ unitär äquivalente Teildarstellung. Wegen der Struktur von $\mathscr{B}$ gibt es einen Hilbertschen Raum $\mathscr{K}_{1}$ und eine stetige irreduzible *-Darstellung $\tau_{1}$ von $\pi\left(C^{*}(G)\right)$ in $\mathscr{K}_{1}$ mit $\mathscr{K}=\mathscr{K}_{1} \otimes V$ und $\tau(a \otimes b)=\tau_{1}(a) \otimes b$ für $a \in \pi\left(C^{*}(G)\right)$, $b \in \mathscr{B}(V)$. Sei nun $\tilde{\tau}_{1}=\tau_{1} \circ \pi$. Dann ist $\tilde{\tau}_{1}$ eine stetige irreduzible *-Darstellung von $C^{*}(G)$, und nach Konstruktion gilt:

$$
\tilde{\tau}_{1} \ll \pi \text {. }
$$

Ferner verifiziert man leicht die Formel

$$
\left.\tau\right|_{\infty} \circ \pi \otimes \varrho=\tilde{\tau}_{1} \otimes \varrho \text {. }
$$

Wir schränken nun $\tau$ und $\pi^{\prime}$ auf $(\pi \otimes \varrho)\left(C^{*}(N)\right)=\pi\left(C^{*}(N)\right) \otimes 1_{V} \subseteq \mathscr{A} \subseteq \mathscr{B}$ ein. Aus der Tatsache, daß $\pi^{\prime}$ in $\left.\tau\right|_{\mathscr{A}}$ enthalten ist, ergibt sich dann, daß $\left.\pi\right|_{N}$ in $\left.\bar{\tau}_{1}\right|_{N}$ enthalten ist. Nach Lemma 2 gibt es eine irreduzible unitäre Darstellung $\alpha$ von $G / N$ mit

$$
\tilde{\tau}_{1} \sim \pi \otimes \alpha .
$$

Da $\pi^{\prime}$ in $\left.\tau\right|_{\mathscr{A}}$ enthalten ist, ist $\pi=\pi^{\prime} \circ \pi \otimes \varrho$ enthalten in $\left.\tau\right|_{\mathscr{A}} \circ \pi \otimes \varrho=\tilde{\tau}_{1} \otimes \varrho \sim$ $\sim \pi \otimes \alpha \otimes \varrho$. Nach der Eindeutigkeitsaussage in Lemma 2 ist dann die triviale Darstellung in $\alpha \otimes \varrho$ enthalten. Folglich ist $\alpha \sim \varrho^{*}$ und mit (1) und (3) ergibt sich die Behauptung.

Beweis des Korollars zu Lemma 3. Nach Lemma 3 ist $\pi \otimes \varrho^{*} \ll \pi$. Da schwaches Enthaltensein beim Tensorieren mit einer festen Darstellung erhalten bleibt (siehe [4]), gilt $\pi \otimes \otimes^{n} \varrho^{*} \ll \pi$ für jede natürliche Zahl $n$. Bekanntlich ist jede 
irreduzible Darstellung einer endlichen Gruppe in einer geeigneten Tensor-Potenz einer festen treuen Darstellung enthalten (vgl. etwa [5], p. 519). Nun braucht $\varrho^{*}$ natürlich nicht treu zu sein, aber $\varrho^{*}$ hat denselben Kern wie $\varrho$ (Kern in $G$, nicht in $C^{*}(G)$ ), und es gibt daher ein $n$ derart, daß $\varrho$ in $\otimes \varrho^{*}$ enthalten ist. Also ist $\pi \otimes \varrho \ll \pi$, w.z.b.w.

Beweis von Satz 1. Wir führen den Beweis durch vollständige Induktion über die Ordnung von $G / N$. Der Induktionsanfang $G=N$ ist trivial. Als nächstes untersuchen wir den Fall, daß der Stabilisator $H$ von $\lambda$ im unitären Dual $N$ von $N$ eine echte Untergruppe von $G$ ist. Da $\lambda$ in $\left.\sigma\right|_{N}$ enthalten ist, gibt es eine irreduzible Teildarstellung $\sigma_{1}$ von $\left.\sigma\right|_{H}$ derart, da $\lambda \lambda$ in $\left.\sigma_{1}\right|_{N}$ enthalten ist. Nach [2] ist $\left.\left.\sigma\right|_{H} \ll \tau\right|_{H}$. Es gibt folglich eine irreduzible Teildarstellung $\tau_{1}$ von $\left.\tau\right|_{\theta}$ mit $\sigma_{1} \ll \tau_{1}$. Da nach Frobenius $\tau$ in ind $\lambda$ enthalten ist, ist $\left.\tau_{1}\right|_{N}$ in $\left.\left(\begin{array}{l}\text { ind } \lambda \\ N \uparrow G\end{array}\right)\right|_{N}$ enthalten, und $\left.\tau_{1}\right|_{N}$ zerfällt in Konjugierte der Darstellung $\lambda$. Mit $\sigma_{1} \ll \tau_{1}$ gilt auch $\left.\left.\sigma_{1}\right|_{N} \ll \tau_{1}\right|_{N}$. Es gibt daher ein $g \in G$ derart, daß $\lambda^{g}$ in $\left.\tau_{1}\right|_{N}$ enthalten ist und daß $\lambda \ll \lambda^{g}$ gilt. Wegen der Endlichkeit von $G / N$ ist dann aber auch $\lambda^{g} \ll \lambda$. Auf Grund der Stetigkeit der Induktion gilt ind $\lambda^{g} \ll$ ind $\lambda$. Da $\tau_{1}$ nach Frobenius in ind $\lambda g$ enthalten ist, gibt es eine irreduzible $N \uparrow H \quad N \uparrow E$ $N \nmid H$

Teildarstellung $\sigma^{\prime}$ von ind $\lambda$ mit $\tau_{1} \ll \sigma^{\prime}$. Wir haben $\sigma_{1} \ll \tau_{1} \ll \sigma^{\prime}$ und daher $N \uparrow H$ ind $\sigma_{1} \ll$ ind $_{H \uparrow G} \tau_{1} \ll$ ind $_{H \uparrow G} \sigma^{\prime}$. Nach Induktionsvoraussetzung $\left(\left.\sigma_{1}\right|_{N}\right.$ und $\left.\sigma^{\prime}\right|_{N}$ enthalten $\left.\lambda\right)$

sind $\sigma^{\prime}$ und $\sigma_{1}$ und dann auch ind $\sigma_{1}$ und ind $\sigma^{\prime}$ schwach äquivalent. Nun ist aber $\tau$ $B \nmid G \quad H \uparrow G$

in ind $\tau_{1}$ enthalten und daher in ind $\sigma_{1}$ schwach enthalten. Nach Lemma 1 ist ind $\sigma_{1}$ $H \uparrow G$
$H \uparrow G$
irreduzibel, also zu $\sigma$ unitär äquivalent, und wir erhalten $\tau \ll \sigma$,w.z.b.w.

Es bleibt noch der Fall zu untersuchen, daß $G$ die Darstellung $\lambda$ fest läßt. Wir fixieren eine Zerlegung $G=\bigcup_{i=1}^{r} g_{i} N$ mit $g_{1}=e$. Da $\lambda$ unter $G$ fest bleibt, gibt es für jedes $i, 1 \leqq i \leqq r$, einen unitären Operator $T_{i}$ im Darstellungsraum $\mathscr{H}$ von $\lambda$ mit $\lambda\left(g_{i} n g_{i}^{-1}\right)=T_{i} \lambda(n) T_{i}^{*}$ für alle $n \in N$. Die $T_{i}$ sind wegen der Irreduzibilität von $\lambda$ bis auf skalare Vielfache (vom Betrage 1) eindeutig bestimmt. Wir fixieren nun Operatoren $T_{i}$, welche der obigen Beziehung genügen; es gelte $T_{1}=\mathrm{id} \mathscr{H}_{\mathscr{H}}$. Sodann definieren wir eine Abbildung $L$ von $G$ in die Gruppe der unitären Operatoren auf $\mathscr{H}$ durch $L\left(g_{i} n\right)=T_{i} \lambda(n)$ für $1 \leqq i \leqq r$ und $n \in N$. Für $x, y \in G$ ist (wegen der Irreduzibilität von $\lambda$ ) $L(x y)$ ein Vielfaches (vom Betrage 1) von $L(x) L(y)$, d.h. es gilt $L(x y)=m(x, y) L(x) L(y)$, wobei $m$ eine Abbildung von $G \times G$ in die Gruppe $\mathbb{T}$ der komplexen Zahlen vom Betrage 1 ist. Für $m$ gilt:

(i) Sind $x, y \in G$ und ist $x \in N$ oder $y \in N$, so ist $m(x, y)=1$.

(ii) Sind $x, y \in G$ und $n_{1}, n_{2} \in N$, so ist $m\left(x n_{1}, y n_{2}\right)=m(x, y)$.

(iii) $m(x y, z) m(x, y)=m(x, y z) m(y, z)$ für alle $x, y, z \in G$.

Auf dem topologischen Raum $\mathbb{T} \times G$ erklären wir nun eine Gruppenstruktur durch $(s, x)(t, y)=\left(\operatorname{stm}(x, y)^{-1}, x y\right)$. Dadurch wird $G_{1}=\mathbb{T} \times G$ zu einer lokalkompakten Gruppe. Die zweite Projektion $p: G_{1} \rightarrow G$ ist ein stetiger Homomorphismus. Das 
Urbild $p^{-1}(N)=: N_{1}$ ist ein direktes Produkt der Gruppen $\mathbb{T}$ und $N$. Durch $\pi(t, x)=$ $=t L(x)$ ist eine stetige irreduzible unitäre Darstellung von $G_{1}$ in $\mathscr{H}$.definiert. Es seien $\sigma^{\prime}$ und $\tau^{\prime}$ definiert durch $\sigma^{\prime}=\sigma \circ p$ und $\tau^{\prime}=\tau \circ p$; ferner sei eine Darstellung $\lambda^{\prime}$ von $N_{1}$ erklärt durch $\lambda^{\prime}=\left.\lambda \circ p\right|_{N}$. Der Charakter $\chi$ von $N_{1}$ sei definiert durch $\chi(t, n)=t^{-1}=t$. Es gilt $\pi \otimes \operatorname{ind}_{N_{1} \uparrow G_{1}} \chi \sim \underset{N_{1} \uparrow G_{1}}{\sim \text { ind }}\left(\left.\pi\right|_{N_{2}} \otimes \chi\right)=$ ind $_{N_{1} \uparrow G_{1}} \lambda^{\prime}$. Da $\sigma$ und $\tau$ in ind $\lambda$ enthalten sind, sind $\sigma^{\prime}$ und $\tau^{\prime}$ in ind $\lambda^{\prime}$ enthalten. Es gibt daher irreduzible Teil$N_{1} \uparrow G_{2}$ darstellungen $\alpha$ und $\beta$ von ind $\chi$ mit $\pi \otimes \alpha \sim \sigma^{\prime}$ und $\pi \otimes \beta \sim \tau^{\prime}$. Mit $\sigma \ll \tau$ gilt

auch $\sigma^{\prime} \ll \tau^{\prime}$ und daher $\pi \otimes \alpha \ll \pi \otimes \beta$. Daraus folgt (mit [4]) $\pi \otimes \alpha \otimes \alpha^{*} \ll \pi \otimes$ $\otimes \beta \otimes \alpha^{*}$, und es gibt deshalb (die triviale Darstellung ist in $\alpha \otimes \alpha^{*}$ enthalten) eine irreduzible Teildarstellung $\varrho$ von $\beta \otimes \alpha^{*}$ mit $\pi \ll \pi \otimes \varrho$. Nach dem Korollar zu Lemma 3 (angewendet auf $G=G_{1}, N=N_{1}, \beta \otimes \alpha^{*}$ und damit auch $\varrho$ ist trivial auf $N_{1}$ ) gilt $\pi \otimes \varrho \ll \pi$ und dann auch $\pi \otimes \varrho \otimes \alpha \ll \pi \otimes \alpha$. Da nun $\beta$ in $\varrho \otimes \alpha$ enthalten ist, ergibt sich $\pi \otimes \beta \ll \pi \otimes \alpha$ und damit $\tau^{\prime} \ll \sigma^{\prime}$, woraus man ohne Schwierigkeiten $\tau \ll \sigma$ erhält, q.e.d.

Bemerkungen. Lemma 1 und 2 sind im Falle separabler Gruppen wohlbekannt, $\nabla$ gl. [6]; ebenso ist es nach [6] klar, daß man die $G$-stabile Darstellung $\lambda$ (im Beweis von Satz 1) zu einer projektiven Darstellung $L$ von $G$ fortsetzen und dann als gewöhnliche unitäre Darstellung von $G_{1}$ auffassen kann. Wie bereits in [8] festgestellt, kann man aus der Gültigkeit von Satz 1 schließen, daß Prim $(G)$ für eine endlich erzeugte diskrete amenable Gruppe $G$ genau dann ein $T_{1}$-Raum ist, wenn $G$ eine nilpotente Untergruppe von endlichem Index enthält.

\section{Literaturverzeichnis}

[1] J. Drxmier, Les $C^{*}$-algèbres et leurs représentations. Paris 1969.

[2] J. M. G. FeLL, The dual spaces of $C^{*}$-algebras. Trans. Amer. Math. Soc. 94, 365-403 (1960).

[3] J. M. G. FELL, Weak containment and induced representations of groups. Canad. J. Math. 14, $237-268$ (1962).

[4] J. M. G. FELL, Weak containment and Kronecker products of group representations. Pacific J. Math. 13, 503-510 (1963).

[5] B. HUPPERT, Endliche Gruppen I. Berlin 1967.

[6] G. W. MACKEY, Unitary representations of group extensions I. Acta Math. 99, 265-311 (1958).

[7] C. C. Mooke, On the Frobenius reciprocity theorem for locally compact groups. Pacific J. Math. 12, 359-365 (1962).

[8] C. C. Mooke and J. Rosenserg, Groups with $T_{1}$ primitive ideal space. J. Functional Analysis 22, 204-224 (1976).

[9] D. Poguntke, Decomposition of tensor products of irreducible unitary representations. Proc. Amer. Math. Soc. 52, 427-432 (1975).

Anschrift des Autors:

Eingegangen am 23. 10. 1975

Detlev Poguntke

Fakultät für Mathematik

Universität Bielefeld

Kurt-Schumacher-Straße 6

D-4800 Bielefeld 1 